\title{
Solution of optimization problems in mechanical engineering based on use of functional semantic networks
}

\author{
V.M. Pashkevich \\ Belarusian-Russian University, \\ Mogilev, Republic of Belarus \\ pvm@bk.ru
}

\begin{abstract}
The paper aims to investigate the possibility of using functional semantic networks for solving optimization problems in mechanical engineering. The results of the research are based on the application of the methods of the functional semantic network theory, the set theory, the graph theory and classical provisions of the mechanical engineering technology and the theory of machines and mechanisms. The paper describes the methodological principles of solving problems of finding optimal parameters for technical systems by using the structural model in the form of a functional semantic network. The paper offers examples of constructing such networks in designing transmission mechanisms as well as in solving the problem of finding optimal modes of machining. It has been established that functional semantic networks contribute to successful solution of optimization problems in mechanical engineering. Besides, due to functional interrelationships between parameters of engineering systems, application of functional semantic networks can reduce consumption of materials used for reducers by up to $8-22 \%$ and the error of cutting by multipoint tools by up to $21-25 \%$.
\end{abstract}

Keywords - functional semantic networks, optimization in mechanical engineering, mechanical reducers, machining accuracy

\section{INTRODUCTION}

A significant number of objectives of modern mechanical engineering are based on application of an optimizationoriented approach, when a solution minimizing or maximizing the predetermined optimality criterion is chosen from a set of admissible solutions.

Objectives of this kind include, for example, design tasks associated with finding optimal construction of a machine or a mechanism that has minimum weight and overall dimensions [1]. Another example of such objectives is the choice of optimal metal cutting modes on the production equipment which, while ensuring the specified dimensional accuracy, simultaneously provide the best productivity or the lowest production costs [2].

To solve similar problems, both classical methods of mathematical programming (linear, nonlinear, dynamic programming, etc.) and search methods in various combinations of gradient methods or random search methods are used.

\section{THEORY}

The purpose of this paper is to investigate the possibility of using functional semantic networks (FSN) for solving optimization problems in engineering.

Functional semantic networks are based on application of methods of the set theory and the graph theory. To solve the equations involved in the relations of FSN, classical numerical methods (the bisection method, the brute-force algorithm) are used. To process relations of the semantic network, methods of symbolic processing are applied (reverse Polish notation).

FSN are logical schemes describing functional interrelationships between different parameters of technical objects. A functional semantic network can be represented as a bipartite graph whose vertices correspond to parameters and relations and the edges correspond to connections between them [3]. Thus, the set of vertices of the network $V$ is a union of two disjoint subsets: a subset of $P$ parameters (initial, intermediate and output ones) and a subset of $R$ relations, i.e.:

$$
V=P \cup R,
$$

where $R$ is the set of relations defining functional dependencies between parameters in the form:

$$
R_{i}=\left\{f\left(P_{1}, \ldots, P_{j}, \ldots, P_{k}\right)=0\right\},
$$

where $\quad P_{j}$ is the $j$-th element of the set of parameters $P$ of the network

$R_{i}$ is the $i$-th relation of the network, which determines functional dependencies between the parameters $P_{1}, \ldots, P_{j}, \ldots$, $P_{k}$.

An example of this FSN structure is shown in Fig. 1; the vertices in the form of circles represent the network parameters, and the vertices in the form of rectangles describe their relations. Moreover, the relation $R$, which is defined on the set from $k$ attributes (parameters $P_{1}, \ldots, P_{k}$ ), is determined by a subset of the Cartesian product of domains $D_{i}$ (sets of possible values), i.e.:

$$
R\left(P_{1}, \ldots, P_{j}, \ldots, P_{k}\right) \subseteq D_{1} \times \ldots \times D_{j} \times \ldots \times D_{k}
$$


where $P_{j} \in D_{j},(j=1,2, \ldots, k)$.

Any tuple satisfying the relation $R$ is its element which is equivalent to the condition:

$$
\left(P_{1}, \ldots, P_{j}, \ldots, P_{k}\right) \in R
$$

This means that any change in the value of the parameter $P_{j}$ will automatically result in functionally-related changes in other parameters of relations to ensure that condition (2) is met. Thus, the semantic network will select admissible combinations of the parameters $P_{j}$ satisfying the given relation, which does not require the application of the classical design sequence "from input parameters to output parameters". Both "input" and "output" parameters are equivalent attributes of FSN relations, which provides "open architecture" of calculations.

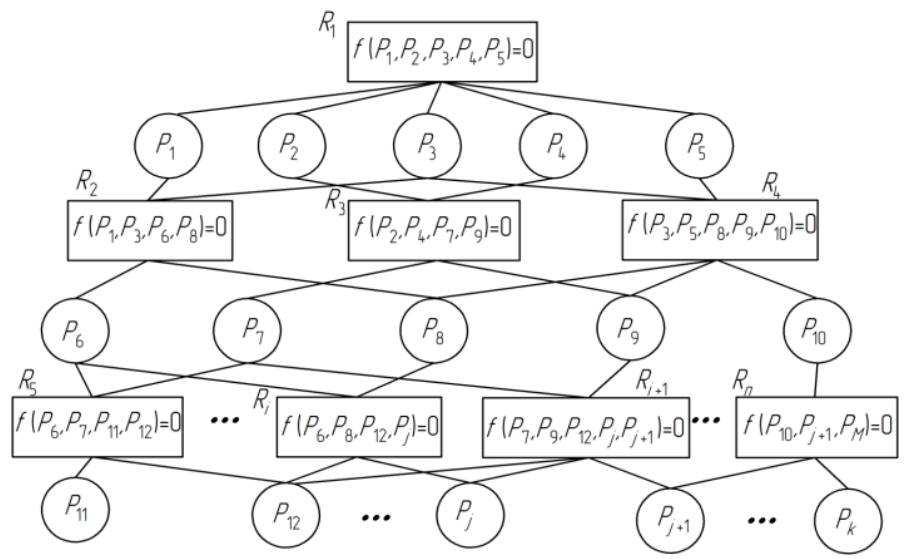

Fig. 1. Example of a functional semantic network.

Thus, for example, if any of the dimensions included in dimensional chains of the structure described in FSN form change, other dimensions of the components of the assembly can be automatically corrected, or, for example, if the material of one of the structural elements changes, its dimensions as well as the dimensions of adjacent parts can be appropriately adjusted to provide the required strength characteristics.

Acceptable values of FSN parameters can be found as follows.

Let us consider relation (2) and determine one of the parameters $P_{j}$ denoting it by $y$. The relationship between the parameters will be functional if the tuples (2) $\left(P_{1}, \ldots, P_{j-1}, P_{j+1}, \ldots, P_{k}\right)$ for the entire set of tuples (2) are different. In this case, no more than one value $P_{j}=y$ corresponds to each tuple $\left(P_{1}, \ldots, P_{j-1}, P_{j+1}, \ldots, P_{k}\right)$ and $\left(P_{1}, \ldots, P_{j}, \ldots, P_{k}\right) \in R$. Thus, the value of $P_{j}=y$ is uniquely determined by the values of the tuple $\left(P_{1}, \ldots, P_{j-1}, P_{j+1}, \ldots, P_{k}\right)$ and corresponds to the function:

$$
y=F^{\left(P_{j}\right)}\left(P_{1}, \ldots, P_{j-1}, P_{j+1}, \ldots, P_{k}\right),
$$

which is called the resolution for the attribute $P_{j}=y$.

Obviously, with one attribute $y$ selected, the rank of relation is equal to one, this relation has one resolution and a one-to-one correspondence is established between the vertices of the semantic network. In this case, the solution corresponds to the tuple $P_{1}, \ldots, P_{k-z}$, which satisfies the set of functions $y_{i}$. The value of the determined attribute can be found by classical methods of numerical solution of the equation, e.g., by the bisection method.

In turn, for the rank of relation $z, z$ functions depending on $k-z$ variables can be determined and $z$ solutions for a minimally closed system of relations can be obtained. As a rule, in this case the algorithms for finding solutions are bruteforce algorithms. In this connection, in practice, to improve the accuracy of the solution and to ensure an acceptable time for finding solutions, only relations with a rank of relation equal to one should be used.

To determine the value of the selected attribute included in the closed system of relations, the relations are transformed into corresponding functions, which corresponds to the transformation of the undirected graph of FSN into a directed graph that describes the input parameters for solving the problem and the output variable which in this case should be determined as follows:

$$
R=\left\{f\left(P_{1}, \ldots, P_{k}\right)=0\right\} \rightarrow F^{\left(P_{j}\right)} \boldsymbol{P}_{1}, \ldots, P_{j-1}, P_{j+1}, \ldots, P_{k} ;
$$

This transformation is illustrated in Fig. 2.
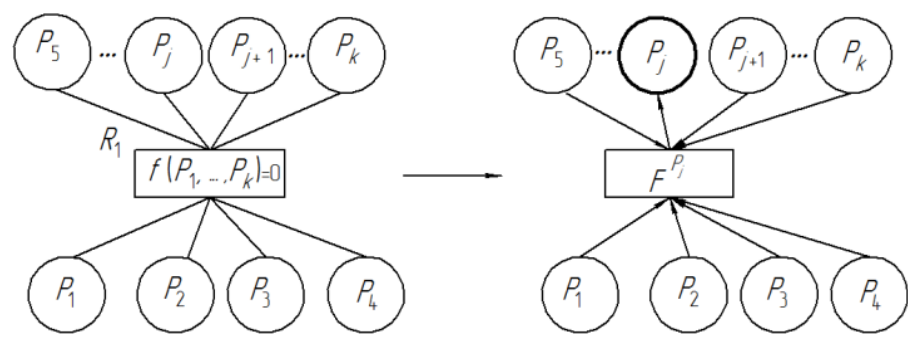

Fig. 2. Example of transformation of a relation into a function.

Similarly, all relations of the system are transformed into corresponding functions, and in order to solve the given problem a sequence of functions is made (Fig. 3).

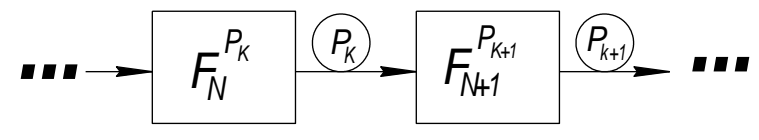

Fig. 3. Generating a sequence of functions.

The described algorithm for finding solution allows organizing the procedure of automated design of mechanisms for any combinations of their input and (or) output parameters or to determine its impossibility provided that the input information is not complete enough to transform the semantic network relations into functions. 
From the point of view of the software implementation of the search procedure, FSN is a knowledge base in which FSN relations and variability intervals of FSN parameters are stored. It is also a shell program that allows searching for a solution by means of this knowledge base. The BelarusianRussian University has developed the intellectual system COMPACT which implements the approach presented above [4]. The search for the solution by the system is based on the use of the syntactic processor module, which independently generates a computation program in relation to the given problem without using a predefined rigid searching algorithm.

This approach has a number of advantages. So, due to understanding the functional interrelations between the parameters of the object the methods of calculating the parameters of machines having a fundamentally different nature can be linked in a common computing environment (e.g., power and kinematic analysis, strength calculations of parts, calculation of dimensional chains, etc.). The design procedure can be determined by the search system without any predefined sequence being prioritized. The structure of search procedures becomes open in this case, since it is not necessary for the system to divide object parameters into "input" and "output" types.

Many engineering optimization problems can be solved by using FSN. Thus, for example, the problem of finding optimal solutions has the following general form:

$$
\begin{aligned}
& \left\{\begin{array}{l}
f_{1}\left(x_{1} ; x_{2} ; x_{3} \ldots x_{n}\right)=a_{1} \\
f_{2}\left(x_{1} ; x_{2} ; x_{3} \ldots x_{n}\right)=a_{2} \\
f_{3}\left(x_{1} ; x_{2} ; x_{3} \ldots x_{n}\right)=a_{3} \\
\ldots \ldots \ldots \ldots \ldots \ldots \ldots \ldots \ldots \ldots \ldots
\end{array}\right. \\
& z=g\left(x_{1} ; x_{2} ; x_{3} \ldots x_{n}\right) \rightarrow \min (\max )
\end{aligned}
$$

where $f_{i}\left(x_{1} ; x_{2} ; x_{3} \ldots x_{n}\right)=a_{i}$ are the problem constraints;

$$
z=g\left(x_{1} ; x_{2} ; x_{3} \ldots x_{n}\right) \text { is the objective function. }
$$

Obviously, the constraints and the objective function can be represented in the form of the following relations:

$$
\begin{gathered}
a_{i}-f_{i}\left(x_{1} ; x_{2} ; x_{3} \ldots x_{n}\right)=0 \\
z-g\left(x_{1} ; x_{2} ; x_{3} \ldots x_{n}\right)=0
\end{gathered}
$$

In this case, the variable $z$ becomes a trigger variable that determines the termination condition for the process of finding the optimal solution (e.g., with decreasing its increments during the last $N$ search cycles to a value less than a predetermined threshold).

\section{RESEARCH FINDINGS AND THEIR DISCUSSION}

On the basis of the described approach the problems of finding the optimal arrangement of the construction are successfully solved [5]. Thus, Fig. 4 shows a schematic of the radial plunger reducer used in the drives of the process equipment, and Fig. 5 shows a fragment of FSN for designing this reducer with the smallest mass and dimensions.

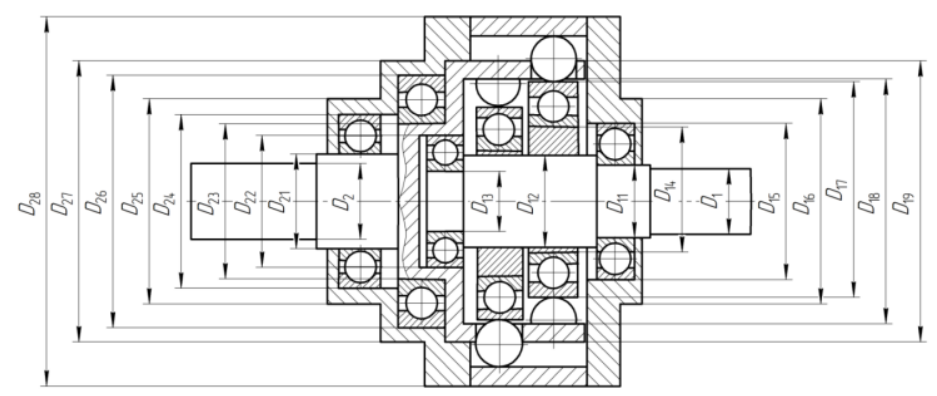

Fig. 4. Schematics of a radial plunger reducer.

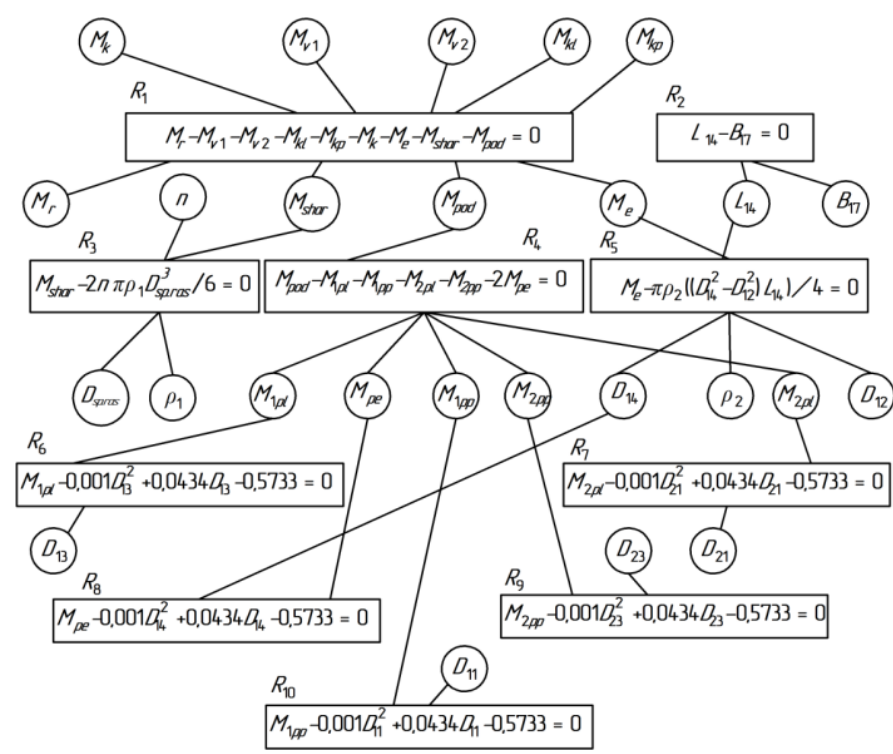

Fig. 5. FSN fragment for designing a radial plunger reducer.

For example, this network takes into account the parameters: $M_{r}$ is the redycer mass; $M_{k l}$ is the left cover mass; $M_{k p}$ is the right cover mass; $M_{V 2}$ is the output shaft mass; $M_{V 1}$ is the drive shaft mass; $M_{e}$ is the eccentric mass; $M_{\text {shar }}$ is the mass of the ball plungers; $M_{p o d}$ is the mass of the bearings; $M_{k}$ is the central wheel mass; $\rho_{1}$ is the density of the ball plungers; $n$. is the number of the ball plungers; $\rho_{2}$ is the density of the eccentric; $M_{1 p l}$ is the mass of the left bearing of the drive shaft; $M_{1 p p}$ is the mass of the right bearing of the drive shaft; $M_{2 p l}$ is the mass of the left bearing of the output shaft; $M_{2 p p}$ is the mass of the right bearing of the output shaft; $M_{p e}$ is the eccentric bearing mass; $B_{17}$ is the width of the eccentric bearing; $D_{s p . r a s .}$ is the diameter of the ball plunger.

The generalized FSN for this reducer includes 66 relations that connect 83 parameters; it makes it possible to solve problems associated with the choice of rational values for parameters that ensure the smallest specific gravity of the reducer. It is established that the described approach, taking into account the functional interrelations between the parameters of the structure, allows reducing the consumption 
of material used for manufacturing the reducer of this type by $8-22 \%$.

The problem of ensuring machining accuracy can also be successfully solved on the basis of FSN [4]. In general, this is a problem of multifactor optimization, the solution of which ensures the maximum machining accuracy, i.e.:

$$
T-\Delta_{\Sigma}\left(\pi_{1}, \pi_{2}, \ldots, \pi_{n}\right) \rightarrow \max
$$

where $T$ is the machining tolerance, $\mathrm{mm}$;

$\Delta_{\Sigma}\left(\pi_{1}, \pi_{2}, \ldots, \pi_{n}\right)$ is the total machining error depending on the parameters of the technological system $\pi_{n}$.

The total machining error can be described by the formula:

$$
\Delta_{\Sigma}=k \sqrt{\varepsilon_{\sigma}^{2}+\varepsilon_{3}^{2}+\varepsilon_{n p}^{2}+\Delta_{y}^{2}}+\Delta_{\Gamma}+\Delta_{T}+\Delta_{c},
$$

where $\varepsilon_{\sigma}$ is the workpiece locating error;

$\varepsilon_{3}$ is the workpiece fixing error;

$\varepsilon_{n p}$ is the error caused by the machine device;

$\Delta_{y}$ is the machining error due to elastic displacements of the tool;

$\Delta_{\Gamma}$ is the machining error due to geometric displacements of the tool;

$\Delta_{T}$ is the error due to thermal deformation of the machine tool;

$\Delta_{c}$ is the error due to inaccuracy, wear and deformation of machines;

$k$ is the empirical factor describing the ratio of the width of the scattered field of the error components to the width of the scattered field described by the normal distribution law.

Further, for each of the above components of the total machining error the decomposition into elementary components is carried out, the corresponding relations are determined and the FSN describing these relations is constructed.

The problem of ensuring accuracy was successfully solved with respect to the accuracy of milling. The knowledge base of this problem included a set of 143 relations that took into account up to 205 parameters of the technological process and equipment. A fragment of this FSN for controlling the machining accuracy for the milling process is shown in Fig. 6.

In the presented parameters and relations of the network, the following notations are used: $u_{0}$ is the tool wear intensity; $l$ is the cutting path; $D_{\text {in }}$ is the tool diameter; $L$ is the estimated length of the cutting with consideration of the tool approach and overtravel; $n$ is the number of parts used for the machine setting between the machine adjustments; $S$ is the tool feed; $\Delta_{\text {доп }}$ is the maximum permissible tool wear; $V$ is the cutting speed; $T$ is the tool life.
For a network described by formula (12), the number of controllable parameters can be from 21 to 89 . It has been established that the application of FSN allows the machining accuracy to be increased to $21-25 \%$ due to moderate cutting modes, optimized machine devices and a number of other organizational solutions (without additional processing equipment, tools, etc.).

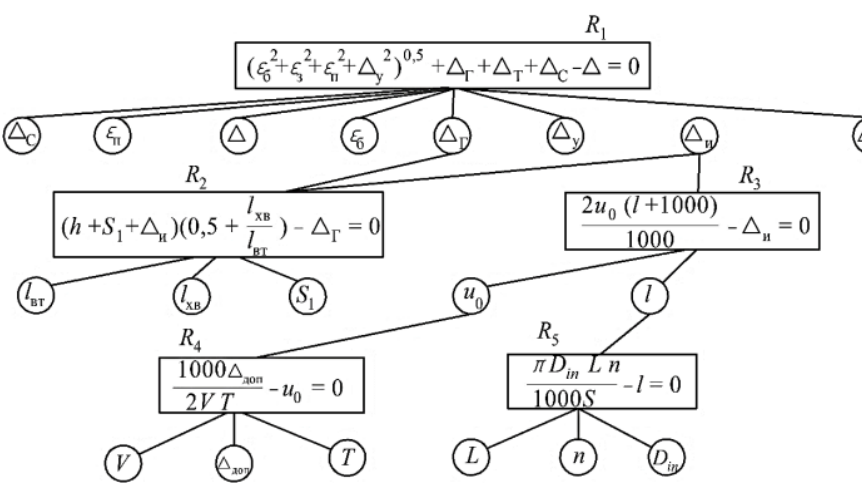

Fig. 6. FSN fragment for providing machining accuracy for the milling process.

\section{CONCLUSIONS}

The results of this study indicate that that FSN are an effective tool for solving optimization problems in which a significant number of parameters functionally interconnected with each other need to be taken into account.

By the example of radial plunger reducers it was established that the use of FSN based on the functional interrelations between the construction parameters leads to reducing the consumption of materials for transmissions of this type by $8-22 \%$ compared to other methods currently in use.

The results of this investigation show that functional semantic networks used to ensure the dimensional accuracy by means of a multipoint tool allow reducing the error of cutting by $21-25 \%$ due to the use of moderate cutting modes, the use of optimized machine devices and a number of other organizational solutions (without additional processing equipment, tools, etc.).

\section{References}

[1] A.R. Parkinson, R.J. Balling, J.D. Hedengren, "Optimization Methods for Engineering Design. Applications and Theory". Brigham Young University, 2013.

[2] F.D.Anania, A.E.Pena, M.Zapciu, "Surface quality and machining time optimization based on federate correction function of tool trajectories types“. Technical gazette, pp. 987-992, 2017.

[3] G.S. Pospelov, "Artificial intelligence is the basis of new information technology“. Moscow, Nauka Publ., 1988.

[4] V.M. Pashkevich, M.N. Mironova, "Functional semantic networks for machining accuracy“. Belarusian-Russian Univ. Publ., 2015.

[5] V.M. Pashkevich, "Methodology of computer-aided design of mechanisms based on the use of functional semantic networks and learning procedure“. Bulletin of the Belarusian-Russian University, no.4, pp. 36-45, 2016. 